\title{
Intratumor stromal proportion predicts aggressive phenotype of gastric signet ring cell carcinomas
}

\author{
Dakeun Lee ${ }^{1} \cdot$ In-Hye Ham $^{2,3} \cdot$ Sang Yong Son ${ }^{2} \cdot$ Sang-Uk Han ${ }^{2} \cdot$ \\ Young-Bae $\mathrm{Kim}^{1} \cdot$ Hoon $\mathrm{Hur}^{2,3,4}$ (B)
}

Received: 8 August 2016/ Accepted: 8 November 2016/Published online: 17 November 2016

(c) The International Gastric Cancer Association and The Japanese Gastric Cancer Association 2016

\begin{abstract}
Objective The aim of this study was to evaluate the prognostic significance of the intratumor stromal proportion in gastric signet ring cell (SRC) carcinomas.

Background Cancer stroma, as exemplified by cancer-associated fibroblasts (CAFs), plays critical roles in cancer proliferation, invasion, and metastasis.

Methods One hundred seventy-five SRC carcinoma cases were classified according to the intratumor desmoplastic stromal proportion to then analyze the clinicopathologic characteristics of stroma-rich cases. We also investigated the impact of CAFs on the migration as well as on the phenotypic changes of gastric SRC carcinomas in vitro.
\end{abstract}

D. Lee and I.-H. Ham contributed equally to this work as co-first authors.

Electronic supplementary material The online version of this article (doi:10.1007/s10120-016-0669-2) contains supplementary material, which is available to authorized users.

Dakeun Lee

dakeun@gmail.com

$\triangle$ Hoon Hur

hhcmc75@naver.com; hhcmc75@ajou.ac.kr

1 Department of Pathology, Ajou University School of Medicine, 164, Worldcup-ro, Yeongtong-gu, Suwon, Gyeonggi-do 16499, Republic of Korea

2 Department of Surgery, Ajou University School of Medicine, Suwon, Republic of Korea

3 Brain Korea 21 Plus Research Center for Biomedical Sciences, Ajou University, Suwon, Republic of Korea

4 Division of Gastrointestinal Surgery, Department of Surgery, Ajou University School of Medicine, 164, Worldcup-ro, Yeongtong-gu, Suwon, Gyeonggi-do 16499, Republic of Korea
Furthermore, we performed RNA sequencing of a pair of CAFs and normal-tissue-associated fibroblasts.

Results Stroma-rich SRC carcinomas (64 of 175 cases, $36.5 \%)$ were associated with female patients $(P=0.045)$, large tumor size $(P=0.007)$, higher $\mathrm{T}$ category $(P<0.001)$, and the presence of perineural invasion $(P=0.018)$. Patients with stroma-rich SRC carcinomas had a significantly shorter disease-free survival $(P<0.001)$ and overall survival $(P<0.001)$. However, in a subgroup analysis, the prognostic significance of the stromal proportion correlated only with patients with T3/4 disease. From multivariate analysis, the high stromal proportion is an independent prognostic factor to predict worse disease-free survival (hazard ratio 2.288; $P=0.001$ ) and overall survival (hazard ratio $2.503 ; P=0.001$ ). We found that CAFs enhanced the migratory abilities of cancer cells through the epithelial-mesenchymal transition, and RNA sequencing results confirmed these findings.

Conclusions The intratumor stromal proportion could be a useful prognostic biomarker and a potential therapeutic target in gastric SRC carcinomas.

Keywords Gastric cancer - Signet ring cell carcinoma . Intratumor stromal proportion - Cancer-associated fibroblast · Prognosis

\section{Introduction}

The bidirectional communication between cells and their microenvironment is important for normal tissue homeostasis. In the same way, cancer cells depend on the stroma, which consists of the extracellular matrix, fibroblasts, endothelial cells, and diverse immune cells (lymphocytes, macrophages, etc.), to sustain their survival [1]. 
A plethora of studies have contributed to characterization of the tumor microenvironment and found that the interactions between cancer cells and their neighboring stroma are critical for cancer progression and metastasis [2, 3]. Recent high-throughput sequencing studies have revealed that cancer is a heterogeneous disease involving aberrant mutations in certain portions of the tumor [4], whereas cancer stroma is also diverse by nature in its composition, stromal proportion, and activation status [5, 6]. Among these, some previous studies focused on the intratumor stromal proportion as a key regulator in cancer biology. A high stromal proportion has been identified as an independent factor associated with poor prognosis in several types of cancers, including esophagus, breast, and colon cancers [7-9].

Invasive cancers frequently have desmoplastic stroma, and the cellular component of the desmoplastic stroma is composed primarily of myofibroblasts, characterized by $\alpha$ smooth muscle actin ( $\alpha$-SMA) expression [10]. These myofibroblasts are generally considered to be cancer-associated fibroblasts (CAFs); they are also known as activated fibroblasts or tumor-associated mesenchymal cells. Unlike normal fibroblasts, CAFs contribute significantly to the events necessary for cancer progression, such as invasion and metastasis, by directly stimulating cancer cell proliferation through the secretion of various growth factors and cytokines, including hepatocyte growth factor, transforming growth factor $\beta$ (TGF- $\beta$ ), and interleukin-6, as well as by remodeling the cancer microenvironment through deposition of extracellular matrix and recruitment of other players such as inflammatory cells and endothelial cells [11-13]. Thus, it is regarded that the aggressive phenotype of cancer is determined, at least in part, by CAFs.

Gastric cancer (GC) is a histologically and genetically heterogeneous disease. Signet ring cell (SRC) carcinoma is a unique histologic subtype of GC, and advanced SRC carcinoma has been considered a more aggressive subtype with propensity for peritoneal dissemination $[14,15]$. We hypothesized that CAFs would impact the progression of gastric SRC carcinoma more than the other histologic subtypes of GC for several reasons. Firstly, gastric SRC carcinoma frequently presents as a "scirrhous" type of cancer on progression, which is associated with abundant desmoplastic (or fibrotic) stroma. Secondly, cancer cells in SRC carcinoma are individually scattered and embedded in the stromal tissue, to possibly gain more opportunities for intimate cross talk between the cancer cells and the surrounding stroma. To address the role of CAFs in gastric SRC carcinomas, we classified the SRC carcinoma cases according to the intratumor desmoplastic stromal proportion through pathologic examination, and correlated the stromal proportion with other clinicopathologic parameters, including patient survival. We also investigated the impact of CAFs on the migration as well as on the phenotypic changes of gastric SRC carcinomas in vitro, and confirmed these findings using RNA sequencing.

\section{Materials and methods}

\section{Patients and tissue samples}

We collected paraffin-embedded tissues of patients with a diagnosis of SRC carcinoma who underwent total or subtotal gastrectomy from January 2005 to December 2008 at Ajou University Hospital. Hematoxylin and eosin stained slides were reviewed by experienced gastrointestinal pathologists to confirm various pathologic parameters, such as histologic subtype and lymphovascular invasion. We adopted the most predominant histologic subtype for the diagnosis. Clinical data were retrieved from the patient medical records. Patients were excluded if they had received preoperative chemotherapy or radiotherapy. Patients who had distant metastasis at the time of surgery were also excluded. In addition, because it was difficult to clarify the stromal proportion in the tumor limited to the mucosal layer (T1a), we selected patients with primary tumors pathologically diagnosed as T1b (submucosal invasion) or higher. Finally, 175 patients were selected for further analysis. Patients in whom stage II or stage III disease was diagnosed pathologically were recommended to have postoperative adjuvant chemotherapy with 5-fluorouracil-based regimens. If the patients experienced recurrence during a follow-up (every 3 or 6 months), second-line regimens based on 5-fluorouracil, platinum, etc, were administered. The median follow-up duration was 72.5 months. The overall survival (OS) was measured from the date of surgery to the date of death or the last follow-up visit. The disease-free survival (DFS) was defined as the time between the date of surgery and detection of a first recurrence or death. The TNM stages were adjusted on the basis of the seventh edition of the AJCC Cancer Staging Manual [16]. This study was conducted in accordance with the code of ethics of the World Medical Association (Declaration of Helsinki) and was approved by the Institutional Review Board of Ajou University Hospital.

\section{Immunohistochemistry and pathologic classification according to the stromal proportion}

Two expert gastrointestinal pathologists (D.L. and Y.-B.K.) who were blind to the conditions selected representative hematoxylin and eosin stained slides from each case and assessed the intratumor desmoplastic stromal proportion. During this process, slides showing areas of mucosal ulcers 
were excluded from the selection, because ulcer-induced fibrosis may be misinterpreted as cancer-associated stroma. In the case of tumor heterogeneity regarding the amount of stroma, slides showing the greatest proportion of stroma were selected. To confirm the exact stromal compartment, we performed immunostaining on the $4-\mu \mathrm{m}$-thick paraffin tissue sections using antibodies against cytokeratin (AE1/ AE3, prediluted; DakoCytomation, Glostrup, Denmark) and $\alpha$-SMA (1:100; Thermo Scientific, Waltham, MA, USA). The immunostaining was performed with a BenchMark XT autoimmunostainer (Ventana Medical Systems, Oro Valley, AZ, US), and the detection was performed with an Ultraview 3,3'-diaminobenzidine detection kit (Ventana Medical Systems).

Finally, cases that satisfied the following criteria were defined as stroma rich: (1) less than $50 \%$ of cancer cells in the tumor and (2) more than $50 \%$ of intervening stroma composed of fibrosis and/or collagen bundles (confirmed by Masson's trichrome and $\alpha$-SMA staining) in the tumor, as previously described $[7,17]$. The remaining cases were classified as stroma poor. A few controversial cases were evaluated with a multihead microscope, and a consensus was reached. Representative cases of stroma-poor and stroma-rich slides are depicted in Fig. 1.

\section{Cell lines}

Human poorly differentiated gastric SRC carcinoma cell lines, SNU-668 and SNU-601 (both from ascites, Mongoloid male patients), were purchased from the Korean Cell Line Bank and were maintained in RPMI 1640 (HyClone Laboratories, South Lagan, UT, USA) supplemented with $10 \%$ fetal bovine serum (FBS), $1 \%$ penicillin, a

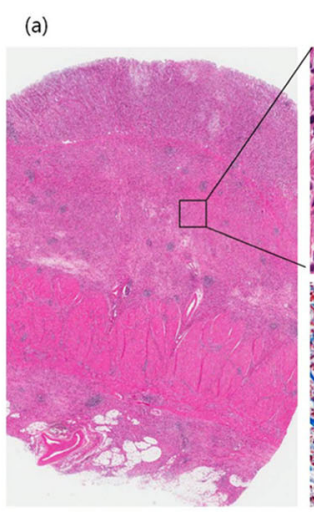

(b)

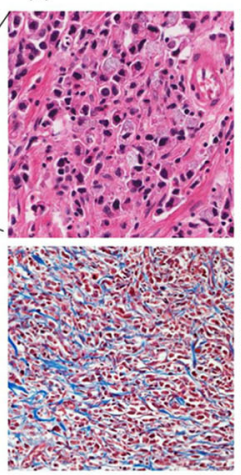

(d) (c)

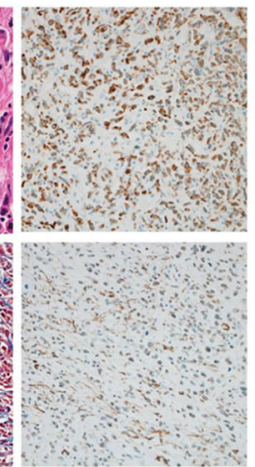

(e)
Fig. 1 Representative photomicrographs of stroma-poor and stromarich gastric signet ring cell (SRC) carcinomas. a Stroma-poor SRC carcinomas show dense cellular infiltration in the entire gastric wall. Only a few intervening stroma are identified; $\alpha$-smooth muscle actin ( $\alpha$-SMA)-positive fibroblasts are also scarce. b Stroma-rich SRC and streptomycin at $37{ }^{\circ} \mathrm{C}$ in a humidified atmosphere containing 5\% $\mathrm{CO}_{2}$. Both cell lines are known to have KRAS mutations [18].

\section{Isolation and culture of fibroblasts}

Stomach cancer specimens were obtained from patients undergoing surgery at Ajou University Hospital (Suwon, Korea) whose preoperative pathologic diagnosis was SRC carcinoma. An experienced pathologist grossly examined and obtained representative samples of the tumor tissues and distal normal tissues. Tissue samples were washed with phosphate-buffered saline with $5 \%$ penicillin and streptomycin. Then, tissues were cut into small pieces and minced with scalpels in a culture dish. Fibroblasts were isolated from both GC tissues (CAFs) and paired normal tissues (normal-tissue-associated fibroblasts, NAFs) (Fig. S1a, b). Isolated fibroblasts were maintained in Dulbecco's modified Eagle's medium (HyClone Laboratories) supplemented with $10 \%$ FBS, $1 \%$ penicillin, and streptomycin. The phenotype of the isolated fibroblasts was confirmed by staining with fibroblast specific protein 1 and vimentin (1:50, Abcam, Cambridge, UK) (Fig. S1c). We only used fibroblasts within six passages.

\section{Co-culture with CAFs or NAFs}

SNU-668 and SNU-601 cells were seeded on the bottom of six-well plates at $10^{5}$ cells per well. Then, NAFs or CAFs were seeded on the upper insert membrane $(0.4-\mu \mathrm{m}$ pore size) of the Transwell chamber (Corning, Union City, CA, USA). The chambers were then inserted into the wells of the plate. Dulbecco's modified Eagle's medium $(2 \mathrm{~mL}$;

b

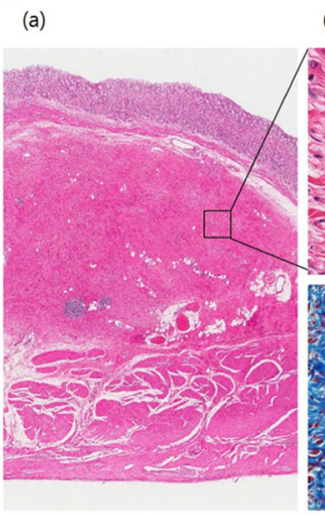

(b)

(c)

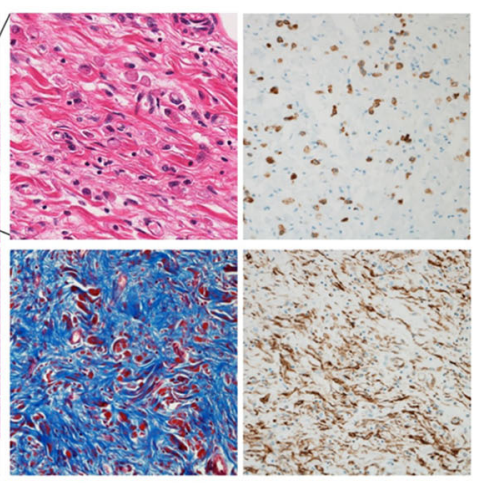

(d)

(e)

carcinomas show dense submucosal fibrosis with only sparse tumor cells. Abundant tumor stroma is found, and numerous $\alpha$-SMApositive fibroblasts are also identified. $a$ hematoxylin and eosin, scanning image; $b$ hematoxylin and eosin, $\times 400 ; c$ pancytokeratin (AE1/AE3), $\times 200 ; d$ Masson's trichrome, $\times 200 ; e \alpha$-SMA, $\times 200$ 
supplemented with 5\% FBS, $1 \%$ penicillin, and streptomycin) was added to both the upper chamber and the bottom chamber, allowing cross talk of the two cell types. Cells were incubated at $37^{\circ} \mathrm{C}$ for $48 \mathrm{~h}$.

\section{Cell migration assay}

In vitro cell migration assays were performed with a 24-well Boyden chamber with a polycarbonate membrane (8.0- $\mu \mathrm{m}$ pore size, Corning). SNU-668 cells were plated at $10^{4}$ cells per well in Transwell inserts with serum-free Dulbecco's modified Eagle's medium. Control medium (10\% FBS), NAFs $\left(10^{3}\right.$ or $10^{4}$ cells per well), or CAFs $\left(10^{3}\right.$ or $10^{4}$ cells per well) were added to the bottom chamber of the Transwell plate and incubated at $37{ }^{\circ} \mathrm{C}$ for $72 \mathrm{~h}$. After cell migration, the inserts were discarded and the upper side of the filter was swabbed to remove the nonmigratory cells. The filters were then fixed in $100 \%$ cool methanol and stained with hematoxylin and eosin for 5 min each. Randomly, three nonoverlapping areas were selected to count the cells that had migrated. All experiments were performed in triplicate.

\section{Cell proliferation assay}

The cell proliferation was measured with a EZ-Cytox cell viability assay kit (Daeil Lab Service, Seoul, Korea). Briefly, cell suspensions $\left(10^{4}\right.$ cells per well) were seeded into each well of a 96-well plate and incubated at $37{ }^{\circ} \mathrm{C}$ for $72 \mathrm{~h}$. Then, after the cells had been incubated with $10 \mu \mathrm{L}$ of EZ-Cytox reagent for $2 \mathrm{~h}$ at $37{ }^{\circ} \mathrm{C}$, the absorbance was measured by spectrophotometry at $450 \mathrm{~nm}$. Each experiment was performed in triplicate.

\section{Western blot Analysis}

After co-culture with NAFs and CAFs, the cells were washed with phosphate-buffered saline and lysed in the lysis buffer. Lysates were centrifuged at 13,000 rpm for $20 \mathrm{~min}$ at $4{ }^{\circ} \mathrm{C}$ and then incubated on ice for $20 \mathrm{~min}$. Protein concentration was determined by the Bradford assay (Bio-Rad, Richmond, CA, USA). Equal amounts of protein from each sample were resolved by sodium dodecyl sulfate-polyacrylamide gel electrophoresis and transferred onto a poly(vinylidene difluoride) membrane (Millipore, Billerica, MA, USA). The immunoblots were blocked by incubation in 5\% skim milk, $25 \mathrm{mM}$ tris(hydroxymathyl)aminomethane- $\mathrm{HCl}$ ( $\mathrm{pH} 8.0), 150 \mathrm{mM} \mathrm{NaCl}, 0.1 \%$ Tween 20 for $1 \mathrm{~h}$ at room temperature. The membrane was then incubated with the following primary antibodies: antibodies against E-cadherin, vimentin, Slug (1:1000; Abcam), and $\beta$-actin (1:5000; Santa Cruz Biotechnology, Dallas, TX, USA) on a shaker overnight at $4{ }^{\circ} \mathrm{C}$. Protein detection was performed with an enhanced chemiluminescence kit (Abclon, Seoul, Korea).

\section{RNA sequencing and Gene Ontology analysis}

Total RNA was extracted from a pair of CAFs and NAFs with RNA extraction reagents (Qiagen, Valencia, CA, USA). RNA libraries were prepared with a TruSeq stranded messenger RNA library preparation kit (Illumina), and RNA sequencing was performed with an Illumina NextSeq 500 system following protocols for $1 \times 75$ bp sequencing provided. Reads for each sample were mapped to the reference genome (human hg19) by TopHat (version 2.0.13). The aligned results were added to Cuffdiff (version 2.2.0) to find differentially expressed genes. To estimate the functions of the differentially expressed RNAs in CAFs compared with NAFs, we performed Gene Ontology analysis using the DAVID web application (https://david.ncifcrf.gov).

\section{Statistical analysis}

Statistical analysis was performed with IBM SPSS Statistics (version 21 for Mac OS X, IBM, Armonk, NY, USA) and GraphPad Prism (version 6.0 for Mac OS X, GraphPad, La Jolla, CA, USA). The correlation between the stromal proportion and the clinicopathologic factors was analyzed by a chi-square test. A survival analysis was performed by the Kaplan-Meier method, and the differences were assessed by the log-rank test. A multivariate Cox regression model was used to evaluate the prognostic significance of variables. For the data from in vitro experiments, the differences among groups were evaluated by one-way ANOVA with post hoc Tukey honest significant difference analysis. Two-sided $P$ values less than 0.05 were considered statistically significant.

\section{Results}

\section{High stromal proportion is correlated with large tumor size and advanced tumor invasion status}

Among the 175 patients with gastric SRC carcinomas, 94 $(53.7 \%)$ were male and $81(46.3 \%)$ were female. The median age was 49 years (range 23-85 years). Tumors were frequently located in the lower third of the stomach ( 78 cases, $44.6 \%$ ), followed by the middle third ( 65 cases, $37.2 \%$ ), and the upper third (32 cases, 18.2\%). Most of the cases were advanced GCs (142 cases, $81.1 \%$ ) and T4 disease was the commonest in this cohort (73 cases, $41.7 \%$ ). One hundred fourteen patients $(65.1 \%)$ had metastatic disease in the regional lymph nodes, and 136 patients (77.7\%) were treated with adjuvant chemotherapy. 
Among the 175 patients with SRC carcinomas, 64 cases $(36.5 \%)$ were classified as stroma rich, whereas the remaining 111 cases $(63.5 \%)$ were considered stroma poor (Table 1). Stroma-rich cases were more frequent in female patients $(P=0.045)$, and also correlated with a large tumor size $(P=0.007)$, a higher T category $(P<0.001)$, and the presence of perineural invasion $(P=0.018)$. However, the patients' age $(P=0.123)$, tumor location $(P=0.213)$ nodal category $\quad(P=0.328), \quad$ or

Table 1 Clinicopathologic characteristics of patients with gastric signet ring cell carcinoma according to the intratumor desmoplastic stromal proportion $(N=175)$

\begin{tabular}{lcccc}
\hline & All patients & $\begin{array}{l}\text { Stroma poor } \\
(n=111)\end{array}$ & $\begin{array}{l}\text { Stroma rich } \\
(n=64)\end{array}$ & $P^{\mathrm{b}}$ \\
\hline Age (years) & & & & \\
$\quad \leq 50$ & 96 & $56(50.5 \%)$ & $40(62.5 \%)$ & 0.123 \\
$>50$ & 79 & $55(49.5 \%)$ & $24(37.5 \%)$ & \\
Sex & & & & \\
Male & 94 & $66(59.5 \%)$ & $28(43.8 \%)$ & 0.045 \\
Female & 81 & $45(40.5 \%)$ & $36(56.2 \%)$ & \\
Tumor size (cm) & & & & \\
$\leq 5$ & 89 & $65(58.6 \%)$ & $24(37.5 \%)$ & 0.007 \\
$>5$ & 86 & $46(41.4 \%)$ & $40(62.5 \%)$ & \\
Location & & & & \\
Upper third & 32 & $18(16.2 \%)$ & $14(21.9 \%)$ & 0.213 \\
Middle third & 65 & $38(34.2 \%)$ & $27(42.2 \%)$ & \\
Lower third & 78 & $55(49.6 \%)$ & $23(35.9 \%)$ & \\
T category & & & & \\
T1b & 38 & $31(27.9 \%)$ & $7(10.9 \%)$ & $<0.001$ \\
T2 & 25 & $22(19.8 \%)$ & $3(4.7 \%)$ & \\
T3 & 39 & $20(18.1 \%)$ & $19(29.7 \%)$ & \\
T4 & 73 & $38(34.2 \%)$ & $35(54.7 \%)$ & \\
N category & & & & \\
N0 & 61 & $41(36.9 \%)$ & $20(31.2 \%)$ & 0.328 \\
N1 & 28 & $19(17.1 \%)$ & $9(14.1 \%)$ & \\
N2 & 26 & $19(17.1 \%)$ & $7(10.9 \%)$ & \\
N3a & 28 & $16(14.4 \%)$ & $12(18.8 \%)$ & \\
N3b & 32 & $16(14.4 \%)$ & $16(25 \%)$ & \\
LVI & & & & \\
Absent & 103 & $66(59.5 \%)$ & $37(57.8 \%)$ & 0.831 \\
Present & 72 & $45(40.5 \%)$ & $27(42.2 \%)$ & \\
PNI & & & & \\
Absent & & & & \\
Present & 100 & & & \\
\hline
\end{tabular}

LVI lymphovascular invasion, $P N I$ perineural invasion

a Staging according to the seventh edition of the AJCC Cancer Staging Manual

b Pearson's chi-square test was used in statistical analyses. Values in italic are statistically significant lymphovascular invasion $(P=0.831)$ was not associated with the intratumor desmoplastic stromal proportion.

\section{High stromal proportion is an independent negative prognostic factor in gastric SRC carcinomas}

The univariate analysis revealed that large tumor size (more than $5 \mathrm{~cm}$ ), higher $\mathrm{T}$ category (T3/4), higher $\mathrm{N}$ category (N2/3), the presence of lymphovascular invasion, and the presence of perineural invasion predicted shorter DFS and OS (Table 2). Additionally, male patients had worse DFS and OS than female patients. The stromal proportion resulted in drastically different clinical outcomes; patients with stroma-rich SRC carcinomas had significantly shorter DFS (66.9 months vs 105.7 months; $P<0.001)$ and OS (61.2 months vs 100.6 months; $P<0.001$ ) (Fig. 2). However, in a subgroup analysis, the DFS and OS of patients with T1b/T2 disease were similar regardless of the intratumor stromal proportion ( $P=0.439$ and $P=0.540$ respectively). In patients with T3/4 disease, high stromal proportion significantly influenced patient survival $(P=0.018$ for DFS, $P=0.008$ for OS). In a multivariate analysis, high stromal proportion (stroma rich) is an independent prognostic factor to predict worse DFS (hazard ratio 2.288; $P=0.001$ ) and OS (hazard ratio $2.503 ; P=0.001$ ), along with tumor size, $\mathrm{T}$ and $\mathrm{N}$ categories, and lymphovascular invasion (Table 3 ). In patients with T3/4 disease, "stroma rich" status is the most powerful prognostic factor to predict worse DFS (hazard ratio 2.575; $P<0.001$ ) and OS (hazard ratio 2.717; $P<0.001)$; its prognostic impact was more significant than that of $\mathrm{T}$ category (T3 vs T4) and that of $\mathrm{N}$ category (N0/1 vs N2/3).

\section{CAFs enhance migration of gastric SRC carcinomas through epithelial-mesenchymal transition}

To determine the impact of CAFs on cancer cell migration and proliferation, we performed a Transwell migration and proliferation assay using the SNU-668 cell line. The migratory ability of cancer cells was minimally increased when they were co-cultured with NAFs or a small amount of CAFs $\left(10^{3}\right)$, but it was remarkably increased by large amounts of CAFs $\left(10^{4}\right)$ (Fig. 3a, b). However, the proliferation activity of cancer cells was not affected by either CAFs or NAFs (Fig. S2). Using Western blot analysis, we observed decreased expression of E-cadherin, but increased expression of vimentin and Slug in response to a CAF coculture, suggesting that CAFs induced epithelial-mesenchymal transition (EMT) of cancer cells (Fig. 3c). Similar changes were noted in response to a NAF co-culture, but the differences were not as marked. 
Table 2 Univariate analyses for overall survival $(O S)$ and disease-free survival $(D F S)$ in 175 gastric signet ring cell carcinoma patients

\begin{tabular}{|c|c|c|c|c|c|}
\hline & Number of patients & OS (months) ${ }^{\mathrm{b}}$ & $P^{\mathrm{c}}$ & DFS (months) ${ }^{b}$ & $P^{\mathrm{c}}$ \\
\hline \multicolumn{6}{|l|}{ Age (years) } \\
\hline$\leq 50$ & 96 & $95.4 \pm 5.3$ & \multirow[t]{2}{*}{0.346} & $91.6 \pm 5.6$ & \multirow[t]{2}{*}{0.216} \\
\hline$>50$ & 79 & $84.8 \pm 5.6$ & & $77.7 \pm 5.9$ & \\
\hline \multicolumn{6}{|l|}{ Sex } \\
\hline Male & 94 & $98.8 \pm 4.9$ & \multirow[t]{2}{*}{0.023} & $78.0 \pm 6.0$ & \multirow[t]{2}{*}{0.030} \\
\hline Female & 81 & $98.8 \pm 4.9$ & & $93.7 \pm 5.4$ & \\
\hline \multicolumn{6}{|c|}{ Tumor size $(\mathrm{cm})$} \\
\hline$\leq 5$ & 89 & $114.6 \pm 3.4$ & \multirow[t]{2}{*}{$<0.001$} & $111.3 \pm 4.0$ & \multirow[t]{2}{*}{$<0.001$} \\
\hline$>5$ & 86 & $66.5 \pm 6.1$ & & $59.0 \pm 6.1$ & \\
\hline \multicolumn{6}{|l|}{ Location } \\
\hline Upper third & 32 & $85.3 \pm 10.0$ & \multirow[t]{3}{*}{0.409} & $74.3 \pm 10.2$ & \multirow[t]{3}{*}{0.252} \\
\hline Middle third & 65 & $87.5 \pm 6.2$ & & $84.1 \pm 6.5$ & \\
\hline Lower third & 78 & $93.9 \pm 5.5$ & & $89.3 \pm 6.0$ & \\
\hline \multicolumn{6}{|l|}{$\mathrm{T}$ category $^{\mathrm{a}}$} \\
\hline $\mathrm{T} 1 \mathrm{~b} / \mathrm{T} 2$ & 63 & $122.9 \pm 1.7$ & \multirow[t]{2}{*}{$<0.001$} & $121.1 \pm 2.4$ & \multirow[t]{2}{*}{$<0.001$} \\
\hline $\mathrm{T} 3 / \mathrm{T} 4$ & 112 & $71.5 \pm 5.2$ & & $64.3 \pm 5.3$ & \\
\hline \multicolumn{6}{|l|}{$\mathrm{N}$ category ${ }^{\mathrm{a}}$} \\
\hline $\mathrm{N} 0 / \mathrm{N} 1$ & 89 & $115.1 \pm 3.7$ & \multirow[t]{2}{*}{$<0.001$} & $112.0 \pm 4.1$ & \multirow[t]{2}{*}{$<0.001$} \\
\hline $\mathrm{N} 2 / \mathrm{N} 3$ & 86 & $66.1 \pm 5.9$ & & $58.3 \pm 5.9$ & \\
\hline \multicolumn{6}{|l|}{ LVI } \\
\hline Absent & 103 & $104.5 \pm 4.3$ & \multirow[t]{2}{*}{$<0.001$} & $99.6 \pm 4.6$ & \multirow[t]{2}{*}{$<0.001$} \\
\hline Present & 72 & $71.7 \pm 6.6$ & & $65.7 \pm 6.9$ & \\
\hline \multicolumn{6}{|l|}{ PNI } \\
\hline Absent & 75 & $113.1 \pm 4.1$ & \multirow[t]{2}{*}{$<0.001$} & $110.2 \pm 4.7$ & \multirow[t]{2}{*}{$<0.001$} \\
\hline Present & 100 & $74.4 \pm 5.6$ & & $67.2 \pm 5.7$ & \\
\hline \multicolumn{6}{|l|}{ Stroma } \\
\hline Poor & 111 & $105.7 \pm 4.5$ & \multirow[t]{2}{*}{$<0.001$} & $100.6 \pm 4.9$ & \multirow[t]{2}{*}{$<0.001$} \\
\hline Rich & 64 & $66.9 \pm 6.3$ & & $61.2 \pm 6.5$ & \\
\hline
\end{tabular}

LVI lymphovascular invasion, $P N I$ perineural invasion

${ }^{\text {a }}$ Staging according to seventh edition of the AJCC Cancer Staging Manual

b Mean survival time \pm standard deviation

c Pearson's chi-square test was used in statistical analyses. Values in italic are statistically significant.

\section{RNA sequencing confirmed the role of CAFs as an inducer of EMT}

As a result of RNA sequencing, we obtained 1604 differentially expressed genes (more than twofold) between CAFs and NAFs. Then, we selected 784 overexpressed genes in CAFs. Among these, we found that 103 genes are functionally "secreted," and the Gene Ontology analysis showed that these genes are involved in cell migration $\left(P=1.68 \times 10^{-9}\right)$, cell motility $\left(P=9.32 \times 10^{-9}\right)$, and cell motion $\left(P=1.31 \times 10^{-7}\right)$, which are the key properties of EMT (Fig. 4).

\section{Discussion}

Although identification of prognostic markers and therapeutic targets is the basic foundation for a comprehensive tailored therapy, there is no such biomarker in gastric SRC carcinomas. In the current study, we classified, for the first time, SRC carcinoma cases according to the intratumor desmoplastic stromal proportion, and found that stromarich cases were correlated with large tumor size, higher $\mathrm{T}$ category, and the presence of perineural invasion. We further revealed that a high intratumor stromal proportion is a strong negative prognostic factor in SRC carcinoma 


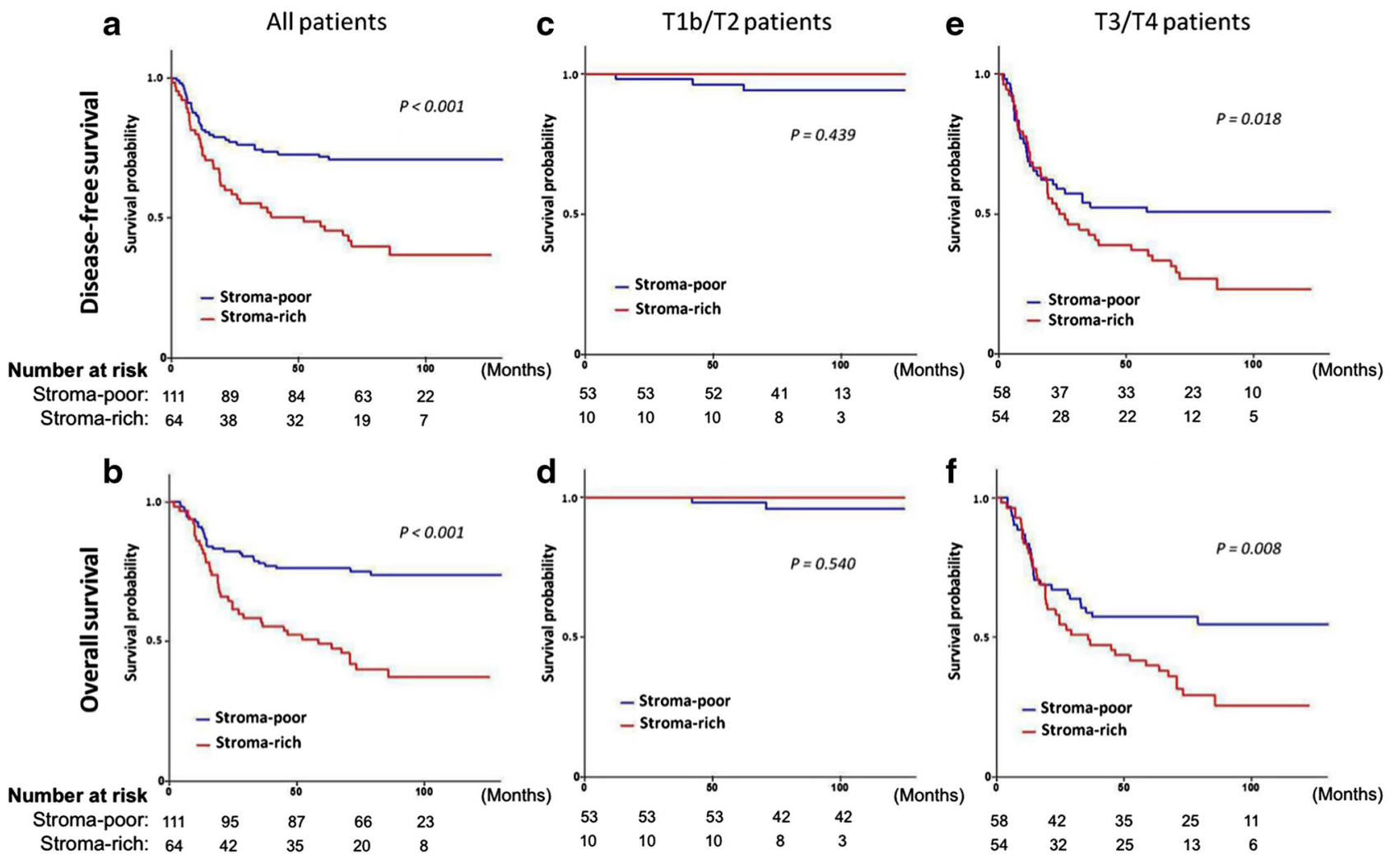

Fig. 2 Kaplan-Meier survival curves of patients with gastric signet ring cell carcinoma. a Disease-free survival (DFS) and b overall survival (OS) curves according to the intratumor stromal proportion

patients. Specifically, the stromal proportion did not influence the prognosis in patients with $\mathrm{T} 1 \mathrm{~b}$ and $\mathrm{T} 2$ cancers, whereas a high stromal proportion was a powerful factor to predict worse prognosis in patients with T3/4 SRC carcinomas. These results indicate that the intratumor desmoplastic stroma (or accumulation of CAFs) increases on progression of gastric SRC carcinoma, and that this stromal portion has significant tumor-promoting roles in gastric SRC carcinomas. Because previous studies have also revealed that a high stromal proportion is an independent prognostic factor in various types of cancers, such as esophagus, breast, liver, and colon cancers [7-9, 17], stromal proportion seems to be a reliable, ubiquitous prognostic factor in malignant epithelial tumors. In addition, because stromal proportion can be easily evaluated and requires no additional cost or facilities beyond standard diagnostics, it would be a major candidate parameter to predict prognosis in patients with SRC carcinoma, especially in patients with $\mathrm{T} 3 / 4$ diseases.

To support the results of our clinical data, we investigated the impact of CAFs that were isolated from advanced gastric SRC carcinoma patients on cancer cells in vitro, and found that CAFs enhanced the migratory abilities of cancer cells through EMT. EMT is a morphogenic process in for the entire 175 patients with signet ring cell carcinoma. Survival curves for $\mathbf{c}$ DFS and $\mathbf{d}$ OS in patients with T1b/T2 disease. Survival curves for $\mathbf{e}$ DFS and $\mathbf{f}$ OS in patients with T3/T4 disease

which cells lose their epithelial characteristics such as cell polarity and cell-to-cell adhesion, and gain mesenchymal properties such as increased cell motility [19]. Although EMT was originally described in its functions during embryonic development, including neural tube formation [20], accumulating evidence has revealed that it plays a critical role in tumor invasion and metastasis, particularly in the process of detachment and migration of cancer cells from the primary tumor and the establishment of metastatic sites in the distant organs [21]. The EMT of cancer cells is often induced by various transcription factors such as Twistl. Sung et al. [22] demonstrated that Twistl was more frequently upregulated in gastric CAFs than in cancer cells, and that increased expression of Twist 1 in CAFs contributed to the progression of cancer cells and poor patient survival. Semba et al. [23] showed that direct interaction with gastric fibroblasts induced EMT-like changes and cancer cell invasion in HSC-39 GC cells. From these findings taken together, CAFs as the major part of the intratumor stroma and the interaction between CAFs and cancer cells appear to have leading roles in the progression of gastric SRC carcinomas by inducing EMT.

Gene expression profiling is a powerful technology providing unbiased information that can be used as a tool in 


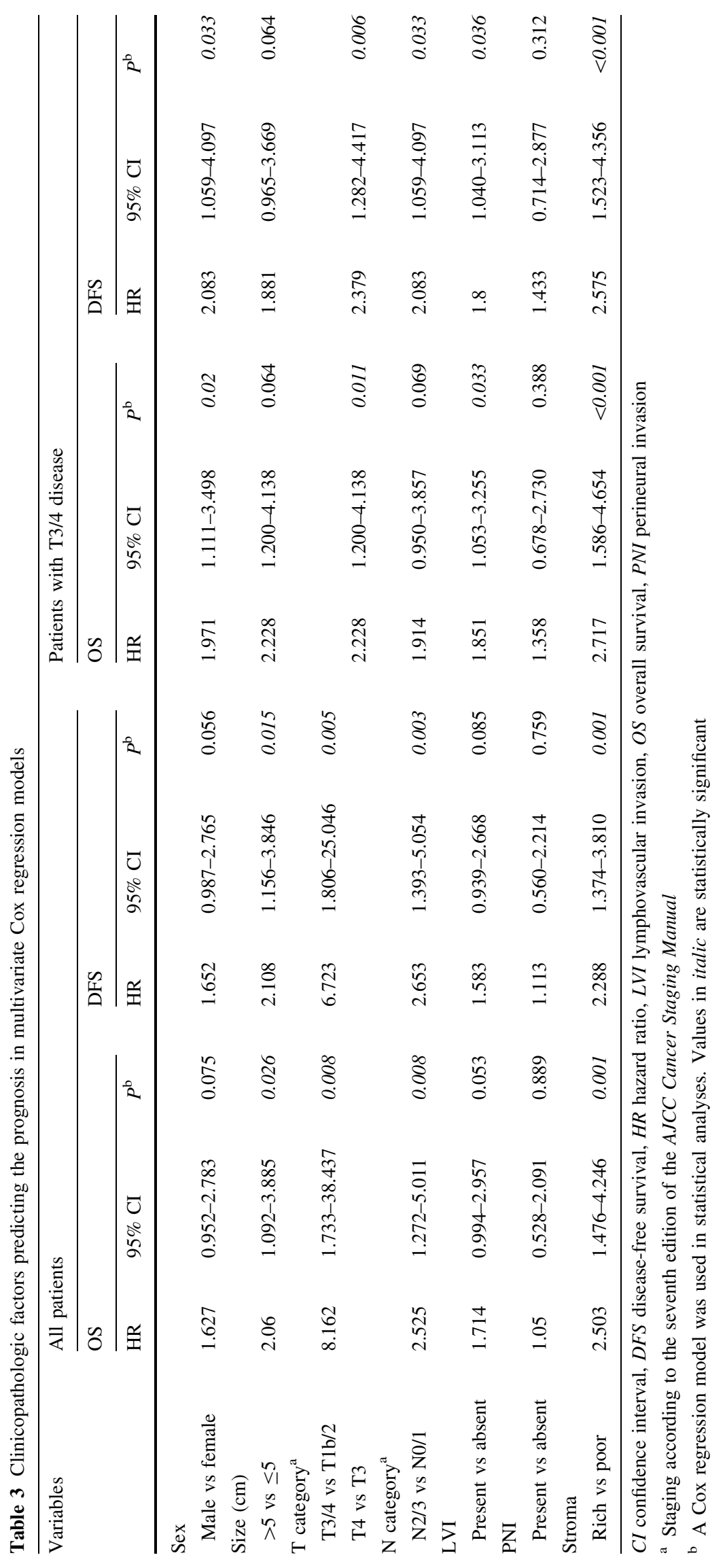



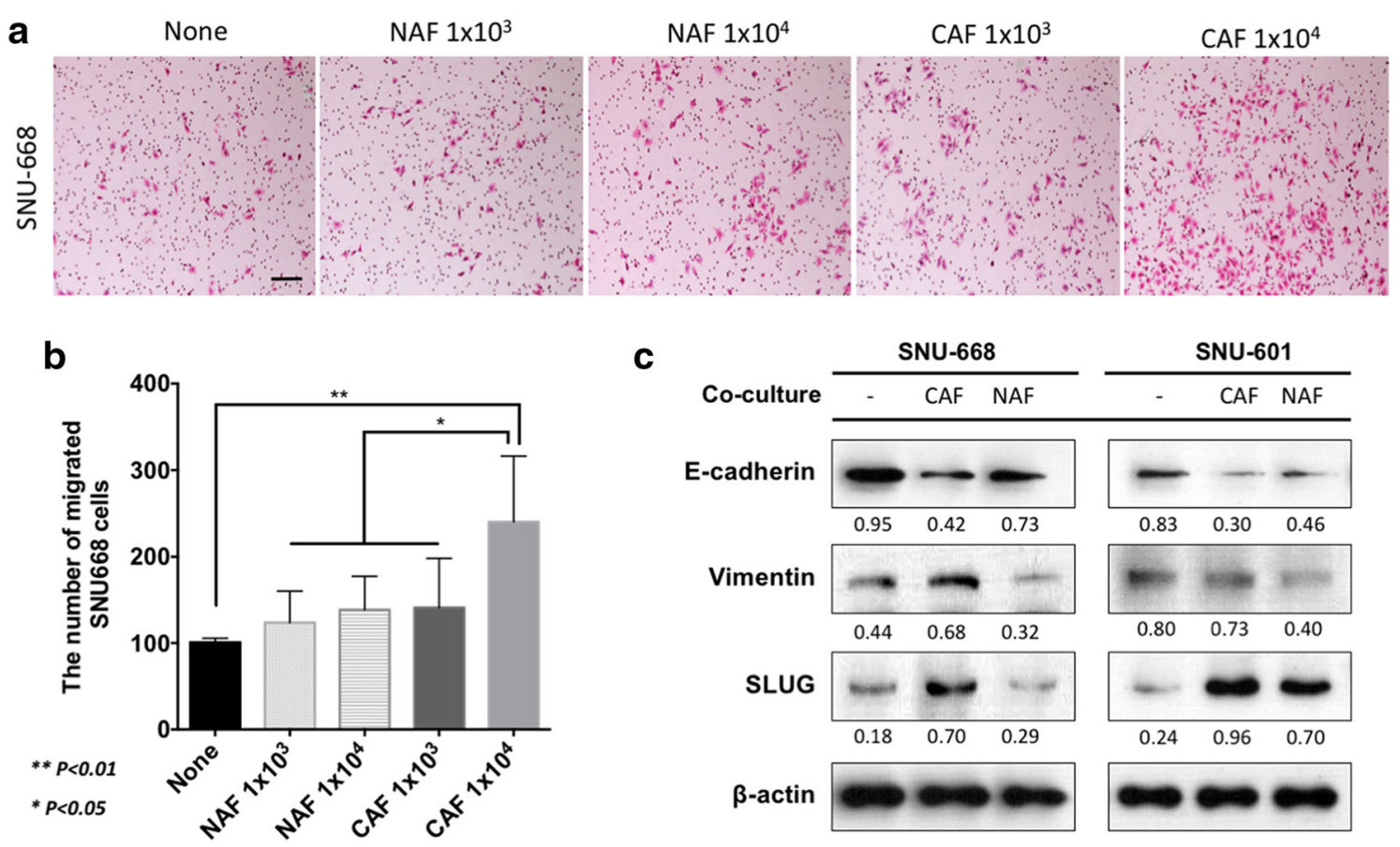

C

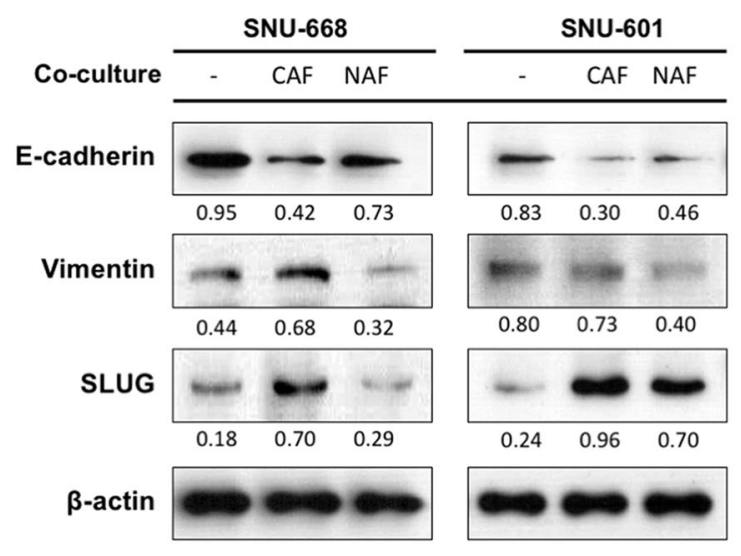

Fig. 3 Effect of cancer-associated fibroblasts on gastric signet ring cell carcinoma cell lines. a, b The number of migrating SNU-668 cells is drastically increased when they are co-cultured with large amounts of cancer-associated fibroblasts. Scale bar $100 \mu \mathrm{m}$. c Reduced expression of E-cadherin and increased expression of vimentin

and Slug in response to co-culture with cancer-associated fibroblasts are observed. The numbers represent the ratio of the expression intensity of the protein relative to $\beta$-actin. $C A F$ cancer-associated fibroblasts, $N A F$ normal-tissue-associated fibroblasts

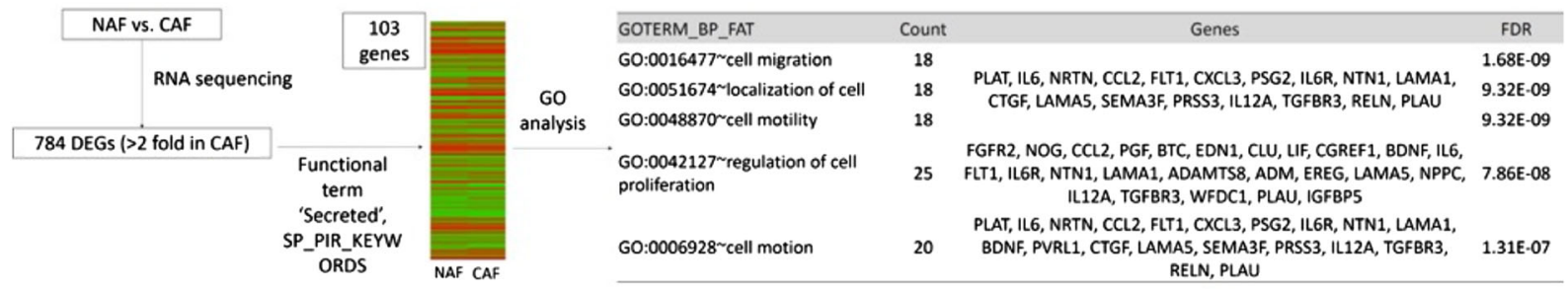

Fig. 4 RNA sequencing results for cancer-associated fibroblasts and normal-tissue-associated fibroblasts show 784 differentially expressed genes (DEGs; more than twofold in cancer-associated fibroblasts). Gene Ontology $(G O)$ analysis reveals that 103 functionally

the classification and prognosis of tumors [24, 25]. Regarding prognosis, many studies have continuously reported that the "stroma signature" is associated with an aggressive clinical course. For examples, Finak et al. [26] used laser-capture microdissection to isolate fibroblasts from normal and breast cancer tissue to establish a "CAF gene signature" consisting of 26 genes, and proved that the CAF gene signature determined poor clinical outcome in four published data sets. Another stroma signature that comprised 278 genes encoding extracellular matrix components was able to stratify breast cancer patients with good and bad prognosis [27]. It was also reported that gene sets defining poor prognosis in colorectal cancer subtypes were predominantly expressed in CAFs. Furthermore, a "secreted" genes are involved in cell migration, cell motility, and cell motion, which are the key properties of epithelial-mesenchymal transition. $C A F$ cancer-associated fibroblasts, $F D R$ false discovery rate, $N A F$ normal-tissue-associated fibroblasts

recent study demonstrated that tumors with a high stromal gene expression in reality contained a high stromal content in GCs [28]. Thus, the proportion of intratumor stroma may serve as a surrogate marker for stromal gene expression levels that have been validated as an excellent prognostic indicator in numerous studies [28, 29], as we demonstrated the prognostic implications of the intratumor stromal proportion in gastric SRC carcinomas in the present study. As previously indicated [28], the intratumor stromal proportion may be used as a potent prognostic marker in histologic subtypes of GC other than SRC carcinomas; however, this needs to be validated in large cohort studies.

Here, the stromal proportion did not influence the clinical outcome of patients with T1b/T2 SRC carcinoma; it 
affected only the prognosis of T3/4 SRC carcinoma patients. The present results cannot be explained by the currently available data. We can only speculate that the properties of CAFs, which can promote cancer progression, depend on a constant cross talk with cancer cells, and thus CAFs may not be formed in the early phase of the disease. In fact, several specific biomarkers determining the characteristics of CAFs, such as Twist-related protein 1, tumor endothelial marker 1 , and lysyl oxidase like 2 , are frequently overexpressed in the fibroblasts of GCs with higher $\mathrm{T}$ categories [22, 30,31], supporting the notion that "fully activated CAFs" may be completed in an advanced stage of disease, with an increased number of CAFs, as shown by our data.

Considering the importance of the tumor microenvironment, many researchers have proposed tumor stroma as a promising target to develop new and effective cancer therapies. In contrast to cancer cells, as the interaction between cancer cells and their environment is probably universal across different tumor types, therapies targeting tumor-microenviroment interactions should be applicable to a large group of patients and they should be less likely to acquire drug resistance because the cancer stroma shows less phenotypic drifts [32]. Currently, TGF- $\beta$ signaling is considered a key regulator in CAF activation or stromal gene expression [28, 29, 33]; therefore, many investigators have tried to inhibit the influence of the cancer stroma using anti-TGF- $\beta$ therapies. Calon et al. [29] showed that the use of TGF- $\beta$ signaling inhibitors to block the cross talk between cancer cells and the microenvironment halted disease progression in patient-derived tumor organoids and xenografts. Currently, a phase 2 clinical trial of LY2157299 (a TGF- $\beta$ receptor 1 specific inhibitor) for the treatment of hepatocellular carcinomas is ongoing, and preliminary results have shown improved clinical outcomes [34]. Because gastric SRC carcinomas are not the usual candidates for the current target agents (trastuzumab or lapatinib), therapies targeting pathways associated with the tumor stroma, such as TGF- $\beta$ signaling, may especially merit consideration in gastric SRC carcinomas.

\section{Conclusions}

We demonstrated that CAFs enhanced the migratory abilities of gastric SRC carcinoma cells by inducing EMT. In clinical samples, we showed that a high intratumor stromal proportion was an independent negative prognostic factor in SRC carcinoma patients with T3/4 disease. Because stromal proportion can be determined easily during routine pathology examinations, these measurements could be a useful and reliable clinical tool for predicting prognosis of gastric SRC carcinoma patients. Furthermore, targeting cancer stroma may be an effective treatment strategy, especially for the treatment of advanced gastric SRC carcinomas.

Acknowledgement This research was supported by the Basic Science Research Program through the National Research Foundation of Korea funded by the Ministry of Education (2014R1A1A2057390 and 2014R1A1A1007905).

\section{Compliance with ethical standards}

Conflict of interest The authors declare that they have no conflict of interest.

Human rights statement and informed consent All procedures followed were in accordance with the ethical standards of the responsible committee on human experimentation (institutional and national) and with the Helsinki Declaration of 1964 and later versions. Informed consent or an appropriate substitute for it was obtained from all patients included in the study.

\section{References}

1. Liu M, Xu J, Deng H. Tangled fibroblasts in tumor-stroma interactions. Int J Cancer. 2011;129:1795-805.

2. Joyce JA, Pollard JW. Microenvironmental regulation of metastasis. Nat Rev Cancer. 2009;9:239-52.

3. Quail DF, Joyce JA. Microenvironmental regulation of tumor progression and metastasis. Nat Med. 2013;19:1423-37.

4. Gerlinger M, Rowan AJ, Horswell S, Larkin J, Endesfelder D, Gronroos E, et al. Intratumor heterogeneity and branched evolution revealed by multiregion sequencing. $\mathrm{N}$ Engl $\mathrm{J}$ Med. 2012;366:883-92.

5. Hanahan D, Coussens LM. Accessories to the crime: functions of cells recruited to the tumor microenvironment. Cancer Cell. 2012;21:309-22.

6. Hanahan D, Weinberg RA. Hallmarks of cancer: the next generation. Cell. 2011;144:646-74.

7. Wang K, Ma W, Wang J, Yu L, Zhang X, Wang Z, et al. Tumorstroma ratio is an independent predictor for survival in esophageal squamous cell carcinoma. J Thorac Oncol. 2012;7:1457-61.

8. de Kruijf EM, van Nes JG, van de Velde CJ, Putter H, Smit VT, Liefers GJ, et al. Tumor-stroma ratio in the primary tumor is a prognostic factor in early breast cancer patients, especially in triple-negative carcinoma patients. Breast Cancer Res Treat. 2011;125:687-96.

9. Mesker WE, Junggeburt JM, Szuhai K, de Heer P, Morreau H, Tanke HJ, et al. The carcinoma-stromal ratio of colon carcinoma is an independent factor for survival compared to lymph node status and tumor stage. Cell Oncol. 2007;29:387-98.

10. Ronnov-Jessen L, Petersen OW. Induction of alpha-smooth muscle actin by transforming growth factor-beta 1 in quiescent human breast gland fibroblasts. Implications for myofibroblast generation in breast neoplasia. Lab Invest. 1993;68:696-707.

11. Marsh T, Pietras K, McAllister SS. Fibroblasts as architects of cancer pathogenesis. Biochim Biophys Acta. 2013;1832:1070-8.

12. Mao Y, Keller ET, Garfield DH, Shen K, Wang J. Stromal cells in tumor microenvironment and breast cancer. Cancer Metastasis Rev. 2013;32:303-15.

13. Cirri P, Chiarugi P. Cancer associated fibroblasts: the dark side of the coin. Am J Cancer Res. 2011;1:482-97. 
14. Piessen G, Messager M, Leteurtre E, Jean-Pierre T, Mariette C. Signet ring cell histology is an independent predictor of poor prognosis in gastric adenocarcinoma regardless of tumoral clinical presentation. Ann Surg. 2009;250:878-87.

15. Chon HJ, Hyung WJ, Kim C, Park S, Kim JH, Park CH, et al. Differential prognostic implications of gastric signet ring cell carcinoma: stage adjusted analysis from a single high-volume center in Asia. Ann Surg. 2016. doi:10.1097/SLA.0000000000001793.

16. Washington K. 7th edition of the AJCC cancer staging manual: stomach. Ann Surg Oncol. 2010;17:3077-9.

17. Lv Z, Cai X, Weng X, Xiao H, Du C, Cheng J, et al. Tumorstroma ratio is a prognostic factor for survival in hepatocellular carcinoma patients after liver resection or transplantation. Surgery. 2015;158:142-50.

18. Qian Z, Zhu G, Tang L, Wang M, Zhang L, Fu J, et al. Whole genome gene copy number profiling of gastric cancer identifies PAK1 and KRAS gene amplification as therapy targets. Genes Chromosom Cancer. 2014;53:883-94.

19. Berx G, Raspe E, Christofori G, Thiery JP, Sleeman JP. PreEMTing metastasis? Recapitulation of morphogenetic processes in cancer. Clin Exp Metastasis. 2007;24:587-97.

20. Duband JL, Monier F, Delannet M, Newgreen D. Epitheliummesenchyme transition during neural crest development. Acta Anat (Basel). 1995;154:63-78.

21. Thompson EW, Newgreen DF, Tarin D. Carcinoma invasion and metastasis: a role for epithelial-mesenchymal transition? Cancer Res. 2005;65:5991-5; discussion 5995.

22. Sung CO, Lee KW, Han S, Kim SH. Twist1 is up-regulated in gastric cancer-associated fibroblasts with poor clinical outcomes. Am J Pathol. 2011;179:1827-38.

23. Semba S, Kodama Y, Ohnuma K, Mizuuchi E, Masuda R, Yashiro $\mathrm{M}$, et al. Direct cancer-stromal interaction increases fibroblast proliferation and enhances invasive properties of scirrhous-type gastric carcinoma cells. $\mathrm{Br} \mathrm{J}$ Cancer. 2009;101:1365-73.

24. Marisa L, de Reynies A, Duval A, Selves J, Gaub MP, Vescovo $\mathrm{L}$, et al. Gene expression classification of colon cancer into molecular subtypes: characterization, validation, and prognostic value. PLoS Med. 2013;10:e1001453.
25. Sadanandam A, Lyssiotis CA, Homicsko K, Collisson EA, Gibb WJ, Wullschleger S, et al. A colorectal cancer classification system that associates cellular phenotype and responses to therapy. Nat Med. 2013;19:619-25.

26. Finak G, Bertos N, Pepin F, Sadekova S, Souleimanova M, Zhao $\mathrm{H}$, et al. Stromal gene expression predicts clinical outcome in breast cancer. Nat Med. 2008;14:518-27.

27. Bergamaschi A, Tagliabue E, Sorlie T, Naume B, Triulzi T, Orlandi R, et al. Extracellular matrix signature identifies breast cancer subgroups with different clinical outcome. J Pathol. 2008;214:357-67.

28. Wu Y, Grabsch H, Ivanova T, Tan IB, Murray J, Ooi CH, et al. Comprehensive genomic meta-analysis identifies intra-tumoural stroma as a predictor of survival in patients with gastric cancer. Gut. 2013;62:1100-11.

29. Calon A, Lonardo E, Berenguer-Llergo A, Espinet E, HernandoMomblona X, Iglesias M, et al. Stromal gene expression defines poor-prognosis subtypes in colorectal cancer. Nat Genet. 2015;47:320-9.

30. Kasashima H, Yashiro M, Kinoshita H, Fukuoka T, Morisaki T, Masuda G, et al. Lysyl oxidase-like 2 (LOXL2) from stromal fibroblasts stimulates the progression of gastric cancer. Cancer Lett. 2014;354:438-46.

31. Fujii S, Fujihara A, Natori K, Abe A, Kuboki Y, Higuchi Y, et al. TEM1 expression in cancer-associated fibroblasts is correlated with a poor prognosis in patients with gastric cancer. Cancer Med. 2015;4:1667-78.

32. De Vlieghere E, Verset L, Demetter P, Bracke M, De Wever O. Cancer-associated fibroblasts as target and tool in cancer therapeutics and diagnostics. Virchows Arch. 2015;467:367-82.

33. Fuyuhiro Y, Yashiro M, Noda S, Kashiwagi S, Matsuoka J, Doi $\mathrm{Y}$, et al. Upregulation of cancer-associated myofibroblasts by TGF- $\beta$ from scirrhous gastric carcinoma cells. $\mathrm{Br} \mathrm{J}$ Cancer. 2011;105:996-1001.

34. Giannelli G, Villa E, Lahn M. Transforming growth factor- $\beta$ as a therapeutic target in hepatocellular carcinoma. Cancer Res. 2014;74:1890-4. 